\title{
libres opinions
}

\section{La radioprotection, c'est aussi de la psychologie}

\author{
A. MOREAU* \\ (Manuscrit reçu le 4 avril 1985)
}

\begin{abstract}
RÉSUME
Radioprotectionniste de terrain dans un centre d'études nucléaires, l'auteur anime également des stages et séances de formation a la radioprotection. II s'interroge ici sur les relations qui existent entre l'opérateur et le spécialiste de radioprotection, sur la nature du travail et sur le rôle que celui-ci peut jouer en sus de son rôle technique, pour contribuer à réduire la dimension émotionnelle, souvent importante, attachée au risque radiologique.
\end{abstract}

\section{ABSTRACT}

Both as a field health physicist at a nuclear research center, and a leader of training sessions in health physics, the author considers the relations between the operator and the health physicist as well as the part the latter can play in order to reduce the highly emotional content of the radiological risk.

\section{RAYONNEMENTS ET PEUR DES RAYONNEMENTS}

Le radioprotectionniste de terrain rencontre dans le concret des problèmes qui sortent des domaines de la physique ou de la biologie : on entre ici dans le domaine des émotions, des attitudes et des opinions.

L'intervention sur des thèmes qui relèvent de la psychologie ou de la psychologie sociale est, en fait, permanente. Le systeme de limitation de dose proposé par la Commission internationale de protection radiologique aborde implicitement ce sujet avec la notion d'optimisation, puisque "toutes les expositions doivent être maintenues au niveau le plus bas que l'on pourra raisonnablement atteindre, compte tenu des facteurs économiques et sociaux" [1].

II suffit de participer à des discussions sur le choix d'une méthode de protection ou d'une procédure opératoire pour comprendre que la détermination du "niveau le plus bas que l'on peut raisonnablement atteindre" fait intervenir des éléments qui ne relèvent pas tous du domaine de la raison [4].

* Commissariat à l'énergie atomique, Centre d'études nucléaires de Saclay, Service de protection contre les rayonnements, 91191 Gif-sur-Yvette Cedex. 
Lors de la préparation d'une intervention ou de l'établissement d'une procédure opératoire, l'agent de radioprotection joue un rôle dans la décision du niveau de l'exposition acceptée, avec les deux autres acteurs : l'opérateur qui subit l'exposition et le responsable hiérarchique qui commande le travail. II en résulte une relation particulière avec ces deux autres acteurs dans ce qui apparaît parfois comme une véritable négociation. Cette relation est à coloration émotionnelle et pas seulement technique - bien que le spécialiste de radioprotection soit officiellement un conseiller technique - puisqu'elle se rapporte au risque encouru par des personnes.

Dans ce cadre, l'agent de radioprotection doit aider l'opérateur à se protéger des rayonnements mais doit également l'aider à "se protéger" des émotions induites par les rayonnements, la peur en particulier.

L'action contre ces émotions est nécessaire pour des raisons de confort de l'opérateur d'abord, car ce sont des émotions désagréables. Mais elle s'impose aussi pour des raisons de sécurité et pour des raisons économiques:

- pour des raisons de sécurité, parce que les émotions, la peur par exemple, peuvent faire "perdre ses moyens" à un opérateur, favoriser les gestes maladroits ou inadaptés ;

- pour des raisons économiques, parce que les émotions deviennent coûteuses lorsqu'elles conduisent à une surprotection technique pouvant entraîner une complication des procédures expérimentales dont le bilan sur le plan de la sécurité n'est pas forcément positif, surtout si l'on prend en compte les conséquences sur la sécurité non radiologique.

\section{LES EMOTIONS EN JEU EN SECURITE RADIOLOGIQUE}

II est intéressant de préciser la nature des émotions en jeu chez l'ensemble des acteurs, en revenant aux tâches de l'agent de radioprotection et en classant celles-ci en trois catégories :

- Il doit contribuer à protéger, rassurer contre ce qui est ressenti comme une agression extérieure particulièrement sournoise puisque non détectable par les sens physiques. Cette agression extérieure est liée au milieu de travail, au propre travail de l'opérateur ou à celui de ses collègues. Cette tâche de protection concerne, en particulier, la mise en place de méthodes techniques de protection et de mesures de rayonnements collectives ou individuelles.

- Il doit conseiller les opérateurs dans leur action, leur apporter éventuellement une aide et une assistance. Cette tâche concerne l'analyse des procédures opératoires prévues, la détermination des consignes, la réalisation de mesures, l'observation sur le terrain aux côtés de l'opérateur du déroulement réel des opérations. Elle concerne également les autorisations de réalisation d'un travail ou de mise en fonctionnement d'une installation.

- Il participe aux décisions collectives concernant le niveau d'irradiation acceptable pour le déroulement des opérations menées par une ou plusieurs personnes. Dans ce cadre, il fait parfois contrepoids, dans une 
optimisation qui ne dit pas son nom, à la tendance d'un responsable de projet polarisé bien naturellement en priorité par la nécessité de réaliser son expérience. La responsabilité de l'agent de radioprotection est ressentie comme étant prépondérante dans le choix collectif du niveau de risque accepté.

Ces trois catégories de tâches se rapportent respectivement :

- à la peur des dangers extérieurs, les rayonnements en l'occurence, risquant de mettre en cause l'intégrité physique ; cette émotion peut aller jusqu'à l'angoisse accompagnée de malaise physique ;

- à la peur, chez l'opérateur, des conséquences de sa propre activité ; il s'agit de la peur de "mal faire", du manque de confiance en soi : "qu'estce que je risque de faire ?" "quelles vont être les conséquences de mes actions ?" "ne vais-je pas être moi-même responsable d'un incident ?". On peut parler, dans certains cas, d'anxiété ; l'application de consignes opératoires, la présence d'une personne spécialiste de la sécurité, qui prend en charge une partie des responsabilités de l'opération jouent alors un rôle rassurant ;

- à la crainte de l'opérateur de se voir imposer un acte présentant un risque, par une décision sur laquelle il n'a pas de prise. II naît alors un sentiment d'impuissance et une inquiétude à l'origine des réactions se formulant par des "où vais-je devoir travailler ?" "que vais-je être obligé de faire?", "quel risque vais-je devoir prendre ?". La situation est alors lourde de ressentiments à l'égard des responsables, des collègues, lourde de conflits entre personnes. Chacun sait, par expérience, l'importance des questions relationnelles sur la sécurité. Les explications fournies par l'agent de radioprotection, son écoute, un travail de mise en relations entre personnes, et d'échanges, sont souvent nécessaires pour cette raison. Le radioprotectionniste est donc aussi un élément de maintien de la cohésion du groupe. Cela est patent en cas d'incident où il est chargé de jouer un rôle anti-panique dans une situation de crise favorable à l'éclatement de ce groupe. Cet aspect de contribution au maintien de la cohésion peut s'observer également dans des installations où l'ambiance n'est, manifestement, pas très bonne. Les agents de radioprotection deviennent alors un élément de stabilité. La sécurité est un thème privilégié d'expression des conflits interpersonnels mais, inversement, peut être le thème sur lequel les personnes recommencent à se parler, en particulier dans des réunions animées par la seule personne extérieure à l'installation qu'est l'agent de radioprotection.

\section{LES RELATIONS ENTRE L'AGENT DE RADIOPROTECTION ET L'OPERATEUR}

Dans ce cadre, les relations entre l'agent de radioprotection et l'opérateur ont bien évidemment une connotation affective et prennent, de façon plus ou moins intense, des aspects ambivalents d'attractiondépendance et de rejet-conflit entremêlés.

L'agent de radioprotection peut être perçu comme celui qui sécurise, qui aide, qui explique, et facilite les relations entre les personnes sur le plan de la sécurité. II en résulte pour lui un statut valorisant et un certain pouvoir qu'il peut être amené inconsciemment à cultiver, si cela permet 
de satisfaire un besoin d'être reconnu, aimé, ou bien d'être craint ou respecté. L'opérateur peut, de son côté, être tenté d'abandonner ce qui concerne sa sécurité dans les mains du spécialiste, se plaçant ainsi, en situation de dépendance.

Au contraire, une situation de conflit peut apparaître. L'opérateur peut reporter sur le spécialiste, dont il a l'impression de dépendre, une part de responsabilité s'il éprouve des sentiments d'insécurité, d'anxiété, devant une tâche à réaliser ou s'il éprouve des sentiments d'impuissance dans le cadre d'une collectivité. II peut aussi refuser, de façon plus ou moins passionnelle, toute relation de dépendance à l'égard d'une personne qui "vient mettre son nez dans ses affaires", une personne dont le statut même le ramène à des émotions, à des inquiétudes qu'il veut nier.

Enfin, l'opérateur peut ressentir l'agent de radioprotection comme une entrave. II existe des comportements consistant à transgresser ostensiblement les règles opératoires et les consignes ou à prendre ostensiblement des risques au vu des autres personnes du groupe. II faut être conscient qu'il s'agit là de formes d'expression, de type réactionnel, masquant la peur du risque, la peur de l'autorité et la crainte de ne pas être suffisamment reconnu par le groupe.

L'impression de contradictions dans l'ensemble de toutes ces attitudes n'est qu'apparente. Sur le terrain de l'affectif, il y a toujours mélange, ambivalence. La surenchère dans les choix techniques peut servir, c'est une tentation, à fuir ce terrain affectif lorsqu'il est ressenti comme incertain. Le spécialiste en sécurité multiplie alors les actes techniques, tel un médecin qui, pour rassurer le patient, se rassurer lui-même et contribuer à des relations sans histoire entre lui et le client, multiplie les actes médicaux et déplace ainsi le problème sur un terrain plus neutre sur le plan relationnel.

\section{LES CONDITIONS D'UNE EVOLUTION - L'EXEMPLE DES STAGES DE FORMATION}

II ne serait pas correct d'accuser les rayonnements d'être à l'origine des émotions. Les émotions se rapportent, en effet, à des besoins fondamentaux de la personne : se sentir en sécurité, maîtriser son travail et le trouver intéressant, se sentir intégré et pris en considération dans une collectivité. Ces besoins sont rarement satisfaits et le risque radiologique sert, en grande mesure, de thème d'expression à l'insatisfaction. L'agent de radioprotection est aussi amené à répondre à des questions, intervenir dans des situations qui ne relèvent que très indirectement de la sécurité radiologique. II est souvent le seul intervenant extérieur au milieu de travail proche, qui écoute, à qui on peut parler. Un objectif possible pour lui est de contribuer à réduire la dimension émotionnelle au moins pour ce qui concerne le risque radiologique, de contribuer à réduire son caractère de thème d'expression pour les émotions dont l'origine est plus générale.

Une réflexion sur la méthode de travail pour atteindre cet objectif s'impose. En ce qui concerne les rayonnements, plusieurs auteurs ont souligné le caractère irrationnel des comportements et des opinions rebelles à une argumentation technique : comparaison des risques radioactifs et des risques classiques, information sur les risques biologi- 
ques réels des rayonnements...[3]. La raison montre ses limites de façon évidente, l'intellect ne suffit pas pour entraîner une évolution.

Une expérience de stages de formation à la radioprotection permet rapidement de convenir des limites de la raison. Des stages de type informatif, de type scolaire traditionnel, où le spécialiste se donne pour seul objectif de transmettre une partie de ses connaissances techniques à des personnes passives, ont pour résultat essentiel d'occulter des interrogations. Certes, de tels stages peuvent satisfaire le formateur qui est demeuré sur le terrain plus sûr des connaissances techniques. Ils peuvent aussi satisfaire les stagiaires qui ont rempli un gros cahier de notes et considèrent que celui-ci leur servira pour faire disparaître leurs craintes. Mais cet effet est temporaire. La situation sur le terrain se retrouve identique à ce qu'elle était auparavant. Les informations techniques sont en grande partie oubliées, d'autres sont utilisées pour conforter les émotions et les opinions pré-existantes.

Les stages de formation à la sécurité radiologique doivent relativiser la dimension intellectuelle au profit d'un travail d'observation des comportements et de reconnaissance des émotions. Pour cela, les stages doivent être centrés sur les stagiaires, sur leurs préoccupations, partir de leur situation, de leur point de vue, en développant l'aspect concret [2]. La reconnaissance des émotions et l'observation des comportements doit porter sur la question des risques et des conditions de travail, mais aussi sur celle des relations avec les agents de radioprotection, les responsables hiérarchiques, et avec les collègues. Ce travail en groupe suppose une attitude de la part de l'animateur qui tranche avec celle d'un simple expert : la dimension d'écoute devient importante. II est nécessaire que le stagiaire se sente dans un climat de confiance pour qu'il puisse aborder des sujets jugés habituellement absurdes ou trop délicats.

Ceci est indispensable pour qu'un stage de radioprotection soit vivant mais tout autant nécessaire dans les échanges quotidiens, dans les réunions de travail ou sur le terrain dans les différentes tâches habituelles. La pratique du radioprotectionniste contribue alors à réduire la dimension émotionnelle attachée au risque radiologique. II en résulte également un changement dans les relations entre l'opérateur et le radioprotectionniste ; celles-ci deviennent moins affectives, ce qui met en cause le modèle " ange gardien" (ange et gardien) du spécialiste en sécurité.

\section{EN CONCLUSION, UN PROGRAMME DE TRAVAIL}

La sécurité radiologique aborde souvent des problèmes techniques complexes, importants. Mais le radioprotectionniste ne doit pas raisonner uniquement en terme de technique. II doit aussi se donner clairement pour objectifs de :

- réduire l'angoisse et l'anxiété, émotions désagréables pour les personnes exposées et travaillant sous rayonnements ;

- améliorer le niveau de Sécurité en favorisant une meilleure maîtrise de la radioprotection par les opérateurs, son intégration dans le processus opératoire ;

- optimiser en limitant les mesures techniques lorsqu'elles sont essentiellement choisies pour rassurer ou pour détendre les relations entre les personnes. 


\section{Pour cela, il est nécessaire d'approfondir des thèmes comme :}

la signification de l'exposition aux rayonnements selon ses diverses modalités pour la situation de travail. Par exemple, le risque de contamination interne est perçu différemment de celui d'irradiation externe [2]. De la même façon, il me semble intéressant de porter attention à la signification du travail de décontamineur, agent d'assainissement radioactif, pour les intéressés eux-mêmes qui l'effectuent, et pour ceux qui profitent de leur travail ;

- la nature des relations qui s'établissent entre les différents acteurs sur le lieu de travail au sujet de la sécurité radiologique : opérateur, spécialiste de la sécurité, responsable fonctionnel, etc. ;

- les conditions, opérations qui favorisent le développement d'une plus grande autonomie de l'opérateur face au risque des rayonnements et l'intégration de la sécurité radiologique dans le processus opératoire ; les attitudes et tâches de l'agent de radioprotection qui permettent cette évolution.

Ces thèmes doivent être abordés par les différents acteurs en groupe. Tout changement ne peut se réaliser, en effet, que s'il est pris en charge, réalisé par les intéressés eux-mêmes, dans un cadre collectif. La dimension d'échange, d'expression, de reconnaissance des émotions rencontrée dans les stages de formation à la radioprotection, lorsqu'ils se déroulent selon la méthode active, doit être développée et peut servir d'exemple pour d'autres structures du type réunion collective de sécurité où l'on trouve l'ensemble des acteurs : hiérarchie, opérateur et radioprotectionniste. Cette dimension se place aussi dans la logique des cercles de qualité et des groupes d'expression dont on parle actuellement.

Le spécialiste de sécurité, en plus de son rôle technique, remplit alors une tâche d'animateur qui facilite l'expression, les rencontres et les échanges. Cela demande des aptitudes ou des aspirations que l'on doit rechercher chez un candidat à un poste de radioprotectionniste. Cela pose, d'autre part, le problème de la formation des techniciens en milieu universitaire et de la formation complémentaire des agents déjà en poste. II ne s'agit pas, bien sûr, d'une formation qui passe par l'intellect mais, là aussi, par la reconnaissance de ses (nos) propres émotions, de ses (nos) propres comportements, de ses (nos) propres motivations lorsque nous remplissons notre rôle de radioprotectionniste.

Ne vivons-nous pas, parfois, de la peur des autres?

\section{BIBLIOGRAPHIE}

[1] COMMISSION INTERNATIONALE DE PROTECTION RADIOLOGIQUE (CIPR). Recommandations... Publications CIPR 26. Oxford: Pergamon Press 1977, p. 3.

[2] FLUCHERE J. Une expérience en matière de formation en radioprotection. Radioprotection 1983, 18, (4), 233-239.

[3] Nucléaire et opinion publique. Revue générale nucléaire 1982, N.2.

[4] SIVADON P., FERNANDEZ A. L'étude des attitudes psychologiques des travailleurs nucléaires vis-à-vis du risque radioactif. Rapport EUR-4198 f, 1968. 